\title{
Bacteroides vulgatus
}

National Cancer Institute

\section{Source}

National Cancer Institute. Bacteroides vulgatus. NCI Thesaurus. Code C86182.

A species of anaerobic, Gram-negative, rod shaped bacteria assigned to the phylum Bacteroidetes. This species is nonmotile, non-sporeforming, hydrolyzes starch and esculin, resistant to penicillin, indole negative, and reduces resazurin, but not neutral red or nitrate. B. vulgatus is found in the human intestinal tract, but can be pathogenic. 